\title{
Prevalencia en el Hospital Español de México de la enfermedad por reflujo gastroesofágico en neonatos pretérmino sintomáticos
}

\author{
Prevalence of gastroesophageal reflux disease in preterm \\ symptomatic neonates at the Hospital Español de México
}

\begin{abstract}
Mario Héctor Blancas Ojeda, ${ }^{*}$ Rubén Martínez Casanova, ${ }^{\ddagger}$ Alejandro Ramón Ángeles Labra, ${ }^{\S}$ Elías Duck Hernández," Laura Baig Tirado," Alejandro Moguel Hernández,** José Edgardo Suárez Morán, ${ }^{\neq \neq}$José Iglesias Leboreiro, ${ }^{\S \S}$ Claudia Isabel Blanco Vela ${ }^{\text {đq }}$
\end{abstract}

\section{Resumen}

Introducción: La enfermedad por reflujo gastroesofágico (ERGE) es común en recién nacidos prematuros. Dicha enfermedad influye en el crecimiento, la duración de la estancia hospitalaria y el resultado respiratorio de estos pacientes. Objetivo: Identificar la frecuencia de ERGE en neonatos pretérmino. Material y métodos: Se incluyeron 58 neonatos a los que se les realizó $\mathrm{pH}$-metría< esofágica de 24 horas, desde enero de 2015 hasta marzo de 2017. Cada neonato presentó tres hallazgos clínicos sugestivos de ERGE (vómitos frecuentes, retraso en el desarrollo y exacerbación de los síntomas respiratorios). El diagnóstico se determinó por una puntuación de Boix-Ochoa $\geq 16.6$. Resultados: Finalmente, $34.5 \%$ de los estudios fue positivo para ERGE $(n=20)$. La mayoría eran varones, es decir, $67.2 \%(n=39)$ con edad media de $33.2 \pm 2.9$. La puntuación de Boix-Ochoa fue de $67.77 \pm 65$. La mayoría de los episodios de reflujo ocurrieron en el periodo postprandial (85\%, N17/20), y en posición supina (70\%, N14/20). El índice de síntomas fue positivo en $38.9 \%(n=7)$ y la probabilidad de asociación de síntomas en $55.6 \%(n=10)$. Conclusión: Existe una asociación entre la prematurez y el diagnóstico de ERGE. La probabilidad de asociación de síntomas es más sensible para el diagnóstico de ERGE en esta población.

Palabras clave: Pretérmino, ERGE, neonato, reflujo.

\section{Abstract}

Introduction: Gastroesophageal Reflux Disease (GERD) is a common problem in preterm newborns. GERD influences growth, length of hospital stays time and the respiratory development. Objective: To identify the frequency of GERD in preterm newborns. Material and methods: We included 58 newborns who underwent $24 \mathrm{hr}$. esophageal pH-metry from January 2015 to March 2017. Each newborn presented 3 suggestive clinical findings for GERD (frequent vomiting, delayed development and exacerbation of respiratory symptoms). Diagnostic was made by a Boix-Ochoa score $\geq 16.6$. Results: Finally, $34.5 \%$ of the studies were positives for GERD $(n=20) .67 .2 \%$ of the patients were male $(n=39)$ with a mean age of $33.2 \pm 2.9$. The Boix-Ochoa score was $67.77 \pm$ $65.85 \%$ of the reflux episodes happened in the postprandial period $(85 \%, \mathrm{~N}=17 / 20), 70 \%$ in supine position $(70 \%, \mathrm{~N}=$ $14 / 20)$. Symptomatic index was positive in $38.9 \%(n=7)$ and the symptoms association probability was $55.6 \%(n=10)$. Conclusions: There is association between prematurity and GERD diagnosis. The GERD symptom association probability is more sensitive in preterm newborns.

Keywords: Preterm, GERD, Neonate, GERD.
* Médico Residente de Pediatría, Facultad Mexicana de Medicina Universidad La Salle.

‡ Servicio de Neonatología, Hospital Español de México.

\$ Servicio de Gastroenterología, Hospital Ángeles Lomas.

I Pediatra-Neonatólogo. Neonatología, Hospital Ángeles Lomas.

" Médico Cirujano M.C., Universidad Anáhuac, México Norte.

** Pediatra-Neonatólogo. Hospital ABC Campus Observatorio.

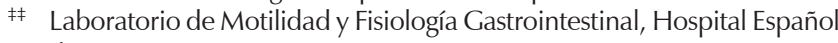
de México.

\section{\$ Pediatra-Neonatólogo. Hospital Español de México. \\ งा Laboratorio de Fisiología Digestiva y Motilidad Gastroinestinal. Servicio de Endoscopia, Hospital Central Militar.}

Correspondencia:

Mario Héctor Blancas Ojeda

Correo electrónico: drblancasojeda@gmail.com

Aceptado: 21-08-2019. 


\section{INTRODUCCIÓN}

La enfermedad por reflujo gastroesofágico (ERGE) constituye uno de los motivos de consulta y de comorbilidades más importantes en la prescripción pediátrica. ${ }^{1}$ Dentro de las enfermedades que requieren tratamiento médico durante el primer año de vida, la ERGE se coloca en el quinto lugar en frecuencia, y es una causa importante de fallo de medro en los primeros tres años de vida. ${ }^{2}$ El reflujo gastroesofágico ocurre comúnmente en el recién nacido y es aún más frecuente en recién nacidos pretérmino, con un porcentaje entre 50 y $60 \%$ de los casos. ${ }^{3}$

La ERGE se define como el paso involuntario del contenido gástrico hacia el esófago; es un reflujo gastroesofágico clínicamente relevante, con altos niveles de morbimortalidad, que repercute de forma directa en el crecimiento ponderal, en el tiempo de estancia intrahospitalaria y en los problemas del tracto respiratorio. ${ }^{4,5}$ El reflujo gastroesofágico ocurre de manera fisiológica entre los recién nacidos; en los lactantes menores y en los recién nacidos prematuros se presenta un riesgo particularmente alto de ocasionar alguna patología. ${ }^{6}$ Los eventos potencialmente letales, como apneas y neumonías por aspiración, cobran mucha importancia en este grupo de edad; en cuanto a las complicaciones agudas y a largo plazo, la falla de medro es la principal complicación que conlleva a una alteración del desarrollo, ${ }^{7}$ que de ocurrir a edades tempranas, provoca consecuencias irreversibles en el crecimiento pondoestatural del paciente. ${ }^{8}$

El principal mecanismo de ERGE en neonatos pretérmino es una relajación transitoria del esfínter esofágico, éste provoca una caída abrupta en la presión del esfínter esofágico inferior, la cual se hace igual o menor a la presión gástrica, sin relación alguna con la motildad esofágica al tragar. 9,10

Algunas de las manifestaciones respiratorias crónicas más frecuentes son: tos, disfonía matutina, apnea, otitis, sinusitis, laringitis, neumonía, sólo por enumerar las más frecuentes. ${ }^{11}$ De igual manera, se asocia con manifestaciones gastrointestinales, intraesofágicas o extraesofágicas, o bien generales (detención del crecimiento, falla en la ganancia de peso, alteraciones en la conducta alimentaria, alteraciones en el patrón del sueño e irritabilidad). ${ }^{12}$ La toma de biopsias por endoscopia en recién nacidos pretérmino está únicamente indicada en pacientes con sospecha de esofagitis y con falla al tratamiento, y que previamente se haya establecido por al menos cuatro semanas. ${ }^{13}$

La pH-metría esofágica de 24 horas es actualmente el estudio diagnóstico estándar de oro para determinar la presencia de enfermedad por reflujo gastroesofágico. ${ }^{14} \mathrm{Su}$ sensibilidad y especificidad son mayores comparadas con los estudios previamente practicados, como la serie esó-

fago-gastroduodenal, que es de operador dependiente. ${ }^{15}$ Se realizan mediciones específicas en el caso de los recién nacidos con la fórmula de Strobel para determinar el sitio apropiado para la colocación de la sonda nasogástrica. ${ }^{16}$ Es necesario entrenar al personal de enfermería a cargo del paciente para reportar los eventos de reflujo (vómitos frecuentes, retraso en el desarrollo y exacerbación de los síntomas respiratorios como apnea, bradicardia, regurgitaciones, retraso en el crecimiento ponderal y exacerbación de síntomas respiratorios) que se presenten durante la realización del estudio, así como su relación con las tomas y la posición del paciente. ${ }^{12}$

No se cuenta con mucha información sobre la enfermedad por reflujo gastroesofágico en pacientes prematuros, por lo que el objetivo de este estudio es el siguiente: a) identificar la prevalencia de ERGE en recién nacidos pretérmino y b) determinar la asociación existente entre ERGE y la edad gestacional.

\section{MATERIAL Y MÉTODOS}

Se trata de un estudio retrospectivo, en el cual se incluyeron de manera consecutiva 58 recién nacidos pretérmino en el Hospital Español de México durante el periodo comprendido entre enero de 2015 y marzo de 2017.

La información clínica, la exploración física y los hallazgos de laboratorios fueron obtenidos por un pediatra a partir de los expedientes clínicos. Dentro de las variables clínicas estudiadas se incluyeron sexo y edad gestacional. Para determinar la presencia de reflujo se utilizó la pHmetría esofágica de 24 horas, la posición del recién nacido (supinación o erecto), la asociación con la alimentación

\section{Tabla 1: Índice de Boix-Ochoa.}

Medición de pH esofágico

Número de episodios por reflujo

Porcentaje de tiempo en que el

$\mathrm{pH}$ es $<4$

Número de episodios $>5$

minutos

Porcentaje de episodios $>5$

minutos

Tiempo promedio de eliminación

de ácido (aclaramiento esofágico)

Episodio de reflujo más largo

Tiempo promedio de eliminación de ácido = (total de minutos en que el $\mathrm{pH}<4) /($ número de episodios de ERGE). ERGE patológico = dos o más criterios anormales. 
Figura 1: Semanas de gestación y ERGE.

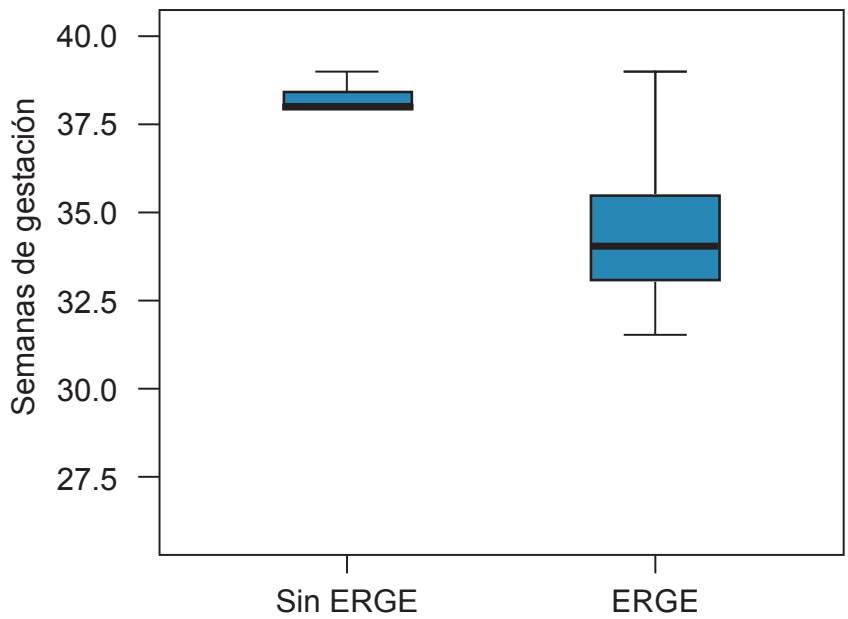

(postprandial o preprandial), el índice de síntomas (positivo o negativo) y la probabilidad de asociación de síntomas (positivo o negativo).

La interpretación de las pH-metrías esofágicas de 24 horas fue realizada por dos gastroenterólogos expertos en fisiología digestiva y neuromotilidad gastrointestinal.

Se definieron como eventos de reflujo en el neonato la presencia de al menos tres hallazgos clínicos sugestivos de ERGE tales como: vómitos frecuentes en cantidad de tres o más al día, regurgitaciones en más de 50\% de las tomas, retraso en el crecimiento y exacerbación de los síntomas respiratorios como tos crónica, apneas y bradicardia.

Como criterios de inclusión se consideraron a todos los recién nacidos pretérmino ( $<37$ semanas de gestación), con pH-metría de 24 horas positiva con base en el índice de Boix Ochoa (Tabla 1), que cumplieran por lo menos con tres hallazgos clínicos (detención del crecimiento, falla en la ganancia de peso, alteraciones en la conducta alimentaria, alteraciones en el patrón del sueño, irritabilidad, tos, disfonía, apnea, otitis y neumonía por aspiración) y que apoyaran el diagnóstico de ERGE. Se excluyeron todos los pacientes pretérmino con necesidad de soporte ventilatorio, ya sea CPAP o intubación endotraqueal; también a pacientes con edad gestacional mayor de 37 semanas, pacientes con $\mathrm{pH}$-metría de 24 horas negativa y pacientes con diagnóstico de reflujo alcalino.

Para la realización de la pH-metría se utilizó un catéter Digitraper MK III S/N 36390, colocado $3 \mathrm{~cm}$ por arriba del esfínter esofágico inferior. La posición del catéter se calculó con la fórmula de Strobel para la inserción nasal de la siguiente forma: longitud esofágica $=5+[0.252 \times$ talla $(\mathrm{cm})]-3$.

El diagnóstico de ERGE se determinó por una puntuación en la escala de Boix-Ochoa $>16.6$. Esta prueba incluye diversos parámetros como el número de episodios de reflujo (NR), el número de episodios de reflujo superior a 5 minutos $(N R>5)$, episodio de reflujo más largo (DR + L) y por último, el índice de reflujo (IR), refiriéndose a la fracción de tiempo con pH medible menor de 4 (Tabla 1).

La escala permite agrupar los resultados positivos en las siguientes tres categorías de acuerdo con el índice de reflujo en: leve $<10 \%$, moderado $10-20 \%$ y grave $>20 \% .^{16}$ La escala de Boix-Ochoa tiene una sensibilidad de $90 \%$ y una especificidad de $100 \%$ en pacientes con síntomas típicos de ERGE.

Los parámetros medidos por este índice se expresan de acuerdo con la edad del recién nacido ( $<15$ días, 24-37 días de vida, 7-8 semanas de vida), lo cual permite establecer con precisión los valores de normalidad específicos para cada población de neonatos.

\section{Análisis estadístico}

Para el análisis estadístico se utilizó el programa SPSS versión 19.0. Las frecuencias se expresaron como porcentajes y como coeficientes de dispersión y se utilizaron tanto la media como la desviación estándar. La comparación estadística entre los grupos se realizó mediante las pruebas de chi cuadrada $\left(\chi^{2}\right)$ y la de t de Student.

\section{RESULTADOS}

Se incluyó un total de 58 recién nacidos pretérmino, de los cuales $67.2 \%$ perteneció al género masculino $(n=39)$. La edad media de la población fue de $33.2 \pm 2.9$ y un total de $34.5 \%(n=20)$ obtuvo una prueba positiva compatible con el diagnóstico de ERGE (Figuras 1 y 2). 70\% (14/20) de la población de neonatos prematuros con ERGE perteneció

Figura 2: Edad media de la población y ERGE.

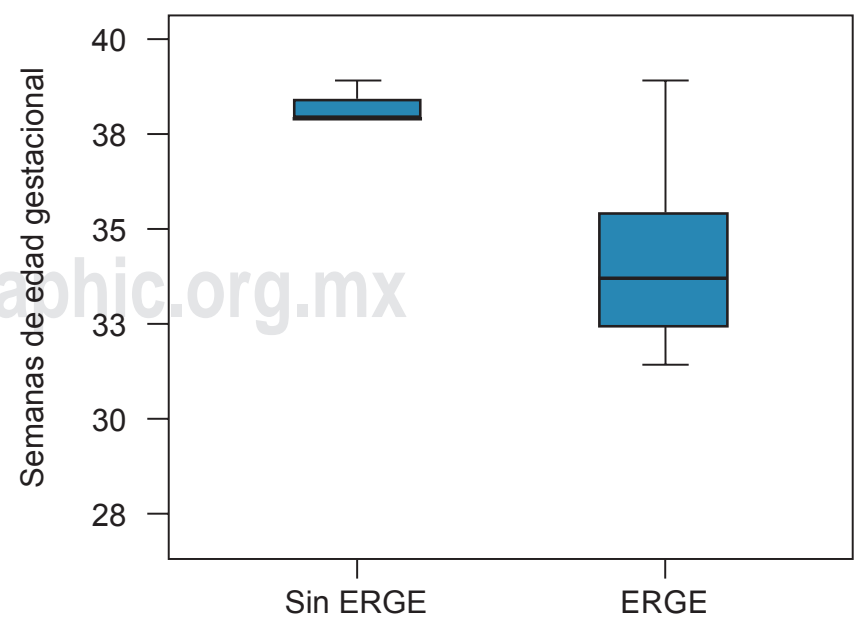


Figura 3: Puntuación de Boix Ochoa.

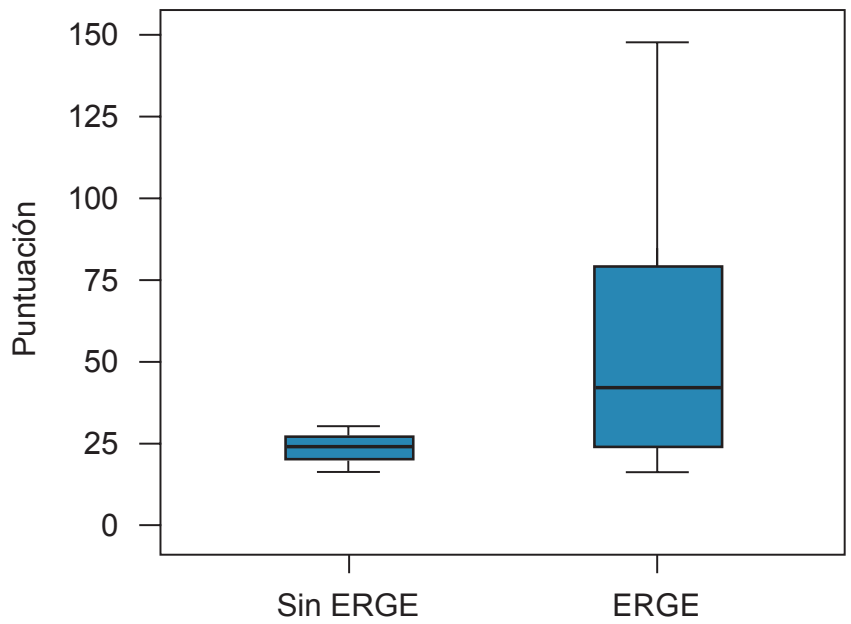

al género masculino y la edad media de los prematuros con ERGE fue de $33.2 \pm 2.9$; la edad media de prematuros sin ERGE fue de $38.3 \pm 0.5$ semanas de gestación $(p<0.001)$.

El valor de la puntuación de Boix-Ochoa fue de $67.77 \pm$ 65 (Figura 3) en aquellos pacientes con estudios positivos. La mayoría de los episodios de reflujo ocurrieron en el periodo postprandial $(85 \%, \mathrm{~N} 17 / 20)$ y en posición supina (70\%, N14/20) (Figuras 4 y 5). El índice de síntomas fue positivo en $38.9 \%(n=7)$ y la probabilidad de asociación de síntomas fue positiva en $55.6 \%(n=10)$. Se observó una asociación significativa entre el sexo femenino y un índice de síntomas positivo, y entre las semanas de gestación y la presencia de ERGE $(p<0.005)$.

Se concluye que existe una asociación significativa entre la prematurez y el diagnóstico de ERGE. La ERGE es más frecuente en neonatos del género masculino. La probabilidad de asociación positiva de síntomas puede ser una herramienta útil para el diagnóstico de ERGE en esta población. Los neonatos prematuros son especialmente susceptibles de presentar apneas o alteraciones en la respiración asociadas con reflujo gastroesofágico, por lo que un diagnóstico oportuno y certero permitirá un tratamiento apropiado y evitará complicaciones.

\section{DISCUSIÓN}

Los recién nacidos pretérmino presentan complicaciones ampliamente estudiadas de acuerdo con las semanas de gestación al momento del nacimiento, así como con los antecedentes perinatales. La enfermedad por reflujo gastroesofágico representa una complicación frecuente en los pacientes prematuros. No existen estudios recientes que representen una realidad clara en cuanto a la distribución y características de esta patología. Dado el aumento en la frecuencia de nacimientos pretérmino y los avances en la medicina para ofrecer tratamiento y mejorar la morbimortalidad de estos pacientes, observar las características de la ERGE resulta un tema de importancia para mejorar el diagnóstico oportuno y la identificación de factores de riesgo, los cuales repercuten directamente en el pronóstico y tiempo de estancia intrahospitalaria de los pacientes.

En este estudio podemos determinar la asociación que existe entre los recién nacidos prematuros y la enfermedad por reflujo gastroesofágico, así como la mayor incidencia en el sexo masculino. Se recaban datos que influyen también en esta enfermedad como la posición del recién nacido (supina) o la relación con el momento de la alimentación (postprandial); estos datos resultan de gran importancia

Figura 4: Asociación de ERGE y posición.

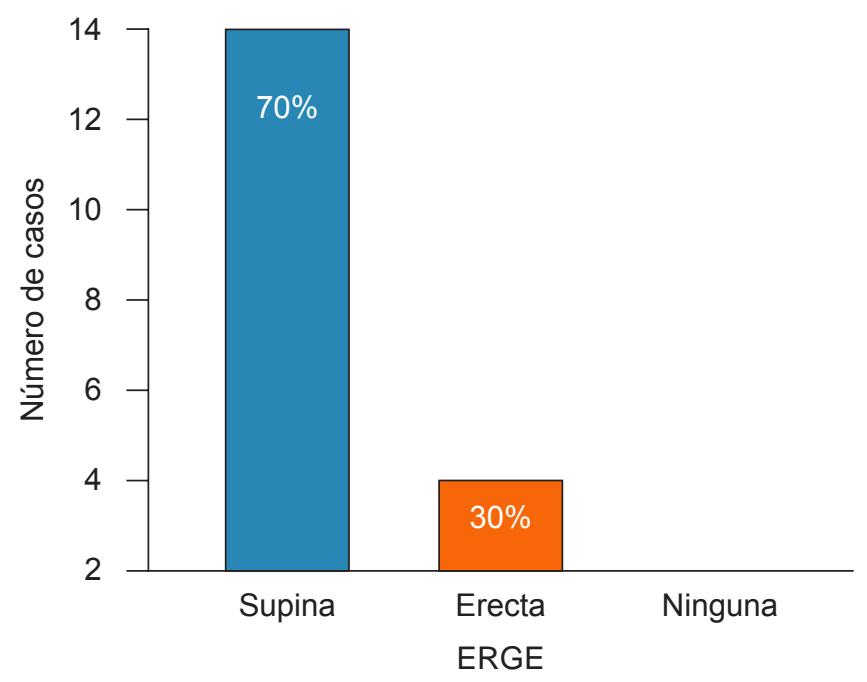

Figura 5: Asociación de ERGE con la alimentación.

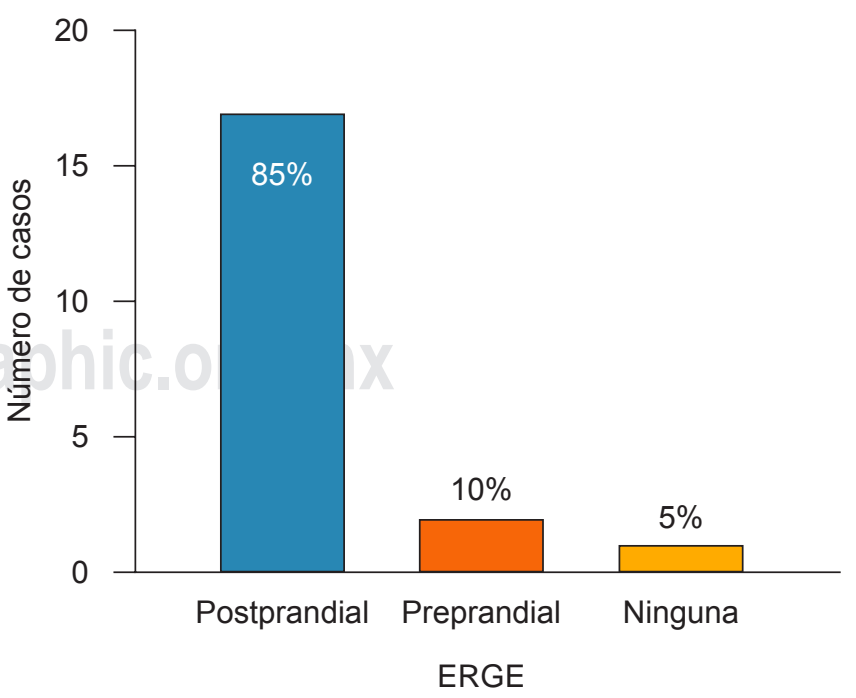


para determinar los factores que influyen en la aparición de esta enfermedad. La información obtenida en este estudio es de gran relevancia, dado que existen muy pocas referencias en cuanto a los valores, escalas y formas de diagnosticar la ERGE en los recién nacidos pretérmino, además de que lleva consigo un alto índice de comorbilidades y aumento en el tiempo de estancia intrahospitalaria. Es fundamental realizar estudios más amplios en este grupo etario para mejorar la atención de los recién nacidos pretérmino.

\section{REFERENCIAS}

1. Rudolph CD, Mazur LJ, Liptak GS, Baker RD, Boyle JT, Colletti RB, Gerson WT, Werlin SL; North American Society for Pediatric Gastroenterology and Nutrition. Guidelines for evaluation and treatment of gastroesophageal reflux in infants and children: recommendations of the North American Society for Pediatric Gastroenterology and Nutrition. J Pediatr Gastroenterol Nutr. 2001; 32 (Suppl 2): S1-31.

2. Sherman PM, Hassall E, Fagundes-Neto U, Gold BD, Kato S, Koletzko S, Orenstein S, Rudolph C, Vakil N, Vandenplas Y. A global evidencebased consensus on the definition of gastroesophageal reflux disease in children. Am J Gastroenterol 2009; 104 (5): 1278-1295.

3. Suárez-Morán E, Morales-Fuentes A, Inzunza-González JA, CedilloLey I, Gerardo-del Hoyo M, Silva- Ramírez H. Influencia de la apnea central del niño prematuro en la enfermedad por reflujo gastroesofágico. Cirugía y Cirujanos. 2001; 79: 511-519.

4. Eichenwald EC, AAP COMMITTEE ON FETUS AND NEWBORN. Diagnosis and Management of Gastroesophageal Reflux in Preterm Infants. Pediatrics. 2018; 142 (1): e20181061

5. Armas Ramos H, Ferrer González JP, Ortigosa Castillo L et al. Reflujo gastroesofágico en niños. Protocolos diagnóstico-terapéuticos de Gastroenterología, Hepatología y Nutrición Pediátrica. Madrid: ERGON; 2010.

6. Michel Penichet F, Ramirez Mayans JA, Montijo Barrios E, Mora Magaña I. Estudios diagnósticos de reflujo gastroesofagico en el paciente prematuro: revisión cualitativa de la literatura. [Tesis]. México: UNAM-INP: 2007. Disponible en: http: //repositorio. pediatria.gob.mx: 8180/handle/20.500.12103/975

7. Jadcherla S, Rudolph C. Gastroesophageal reflux in the preterm neonate. NeoReviews. 2005; 6 (2): e87-e98. DOI: https://doi. org/10.1542/neo.6-2-e87.
8. Vakil N, van Zanten SV, Kahrilas P et al. The Montreal definition and classification of gastroesophageal reflux disease: a global evidencebased consensus. Am J Gastroenterol. 2006; 101: 1900 -1920.

9. Stordal K, Johannesdottir GB, Bentsen BS et al. Gastroesophageal reflux disease in children: association between symptoms and $\mathrm{pH}$ monitoring. Scand J Gastroenterol. 2005; 40: 636-640

10. Rosen R, Vandenplas $Y$, Singendonk M, Cabana M, DiLorenzo C, Gottrand F, Gupta S et al. Pediatric Gastroesophageal Reflux Clinical Practice Guidelines: Joint Recommendations of the North-American Society of Pediatric Gastroenterology, Hepatology and Nutrition (NASPGHAN) and the European Society of Pediatric Gastroenterology, Hepatology and Nutrition (ESPGHAN). J Pediatr Gastroenterol Nutr. 2009; 49: 498-547.

11. Armas Ramos H, Molina Arias M, Peña Quintana L et al. Indicaciones actuales de la monitorización de la pH-metría esofágica. An Esp Pediatr. 2002; 56: 49-56.

12. Lupu VV, Ignat A, Paduraru G, Ciubara A, Moscalu M, Marginean $\mathrm{CO}$, Burlea M. Correlation between the different $\mathrm{pH}$-metry scores in gastroesophageal reflux disease in children. Medicine. 2016; 95 (26): e3804. doi: 10.1097/MD.0000000000003804.

13. van Wijk MP, Benninga MA, Dent J, Lontis R, Goodchild L, McCall LM, Haslam R, Davidson GP, Omari T. Effect of body position changes on postprandial gastroesophageal reflux and gastric emptying in the healthy premature neonate. J Pediatr. 2007; 151: 585-590.

14. Nobile S, Noviello C, Cobellis G, Carnielli VP. Are infants with bronchopulmonary dysplasia prone to gastroesophageal reflux? A prospective observational study with esophageal $\mathrm{pH}$-impedance monitoring. J Pediatr. 2015; 167 (2): 279-285.

15. Cresi F, Locatelli E, Marinaccio C, Grasso G, Coscia A, Bertino E. Prognostic values of multichannel intraluminal impedance and $\mathrm{Ph}$ monitoring in newborns with symptoms of gastroesophageal reflux disease. J Pediatr. 2013; 162 (4): 770-775.

16. Mendes TB, Mezzacappa MA, Toro AA, Ribeiro JD. Risk factors for gastroesophageal reflux disease in very low birth weight infants with bronchopulmonary dysplasia. J Pediatr (Rio J). 2008; 84 (2): 154-159.

Conflicto de intereses: Este estudio no requirió financiamiento alguno y nadie de sus colaboradores presentó ningún tipo de conflicto de intereses.

Patrocinio: Para la elaboración de este trabajo no se recibió ningún patrocinio.

Sponsorship: For the preparation of this work no any sponsorship was received. 